\title{
PROJETO DE LINKS DE FIBRAS ÓPTICAS USANDO TÉCNICAS DE INTELIGÊNCIA ARTIFICIAL
}

\author{
Bruno Cesar dos Santos Lima ${ }^{1}$, Israel Avanci Marques ${ }^{1}$, Nizam Omar ${ }^{2}$ \\ e Rafael Euzébio Pereira de Oliveira ${ }^{1}$ \\ ${ }^{1}$ MackGraphe - Centro de Pesquisas em Grafeno, Nanomateriais e Nanotecnologia - Rua da Consolação, 930, \\ São Paulo, SP, Brasil \\ ${ }^{2}$ FCI - Faculdade de Computação e informática MACKENZIE - Rua da Consolação, 930, São Paulo, SP, Brasil
}

\begin{abstract}
RESUMO
A comunicação digital em nossa sociedade é fortemente baseada em redes interconectadas usando fibras ópticas. Este cenário exige redes melhores com alto desempenho e baixo custo tanto na operação como no projeto da rede. Efeitos lineares e não lineares afetam o desempenho da comunicação em links ópticos e devem ser levados em consider ação no projeto do enlace. $\mathrm{O}$ presente trabalho propõe a utilização de algoritmos de inteligência artificial (IA) para prever distorções de atenuação, dispersão e efeitos não-lineares em projetos de links ópticos, garantindo sua otimização autônoma. Propomos o uso de IA para o projeto de um enlace de fibra óptica dados a distância, taxa de operação e taxa de erro de bits (BER) requerida. Comparamos a performance de 4 algoritmos de IA (redes Bayesianas, redes neurais perceptron (MLP), KNN, e Deep Learning) e obtivemos acurácias de até 99\% e previsão de enlaces otimizados em recursos de amplificação e compensação de dispersão dentro dos parâmetros estabelecidos com a abordagem Deep Learning e KNN.
\end{abstract}

\section{PALAVRAS-CHAVE}

Redes Ópticas, Fibras Ópticas, Inteligência Artificial, Machine Learning, Rede Neural Artificial, Bayes, Deep Learning

\section{INTRODUÇÃO}

Os links ópticos são fundamentais para lidar com a transferência de dados em altas taxas transmitidos através da rede global de internet (Zhao, et al, 2017). Com o crescimento exponencial do tráfego devido ao grande fluxo de dados por vídeos on demand, big data e o advento da internet das coisas (IoT) este cenário tem se agravado (Kyounghyun, et al, 2015), (Shamsuddin, et al, 2015), levando à expansão dos links ópticos existentes e aumentando a demanda por projetos inteligentes de enlaces. A inteligência artificial tem se mostrado uma ferramenta poderosa na solução de problemas em diversas áreas, desde medicina até carros autônomos (Huang, et al, 2004) e com a evolução de componentes de hardware, tem se tornado uma tecnologia promissora e essencial para resolução de desafios científicos complexos.

A pesquisa atual em inteligência artificial aplicada à comunicação óptica conseguiu compensar alguns problemas em enlaces ópticos tais como: gestão dinâmica de amplificadores a fibra dopada com Érbio (EDFAs) (Huang, et al, 2016), monitoramento de desempenho (Wu, et al, 2010) e mitigação de efeitos não-lineares (Gonzalez, et al, 2010). Poucas pesquisas nessa área cobrem o tópico de prever esses efeitos de distorção e fornecer informações para evitar ou diminuir tais distorções no projeto de rede, minimizando os custos de projeto e implementação da rede. Como os algoritmos de machine-learning são extremamente rápidos e podem fazer previsões complexas em poucos minutos, eles possibilitariam a realização de um grande volume de projetos de link ópticos otimizados em um curto espaço de tempo quando comparado ao tempo requisitado por humanos, reduzindo assim seus custos. Esse tipo de solução era inviável anos atrás, pois a automação se limitava a resolver problemas de tarefas repetidas que não exigiam cognição. Entretanto, as técnicas de inteligência artificial vêm se consolidando nessa tarefa de automatizar a resolução de problemas que envolvem lógica e heurística (Tan, et al 2010). O subcampo da inteligência artificial conhecido como "machine learning" tem como vantagens quando comparado à programação clássica o fato de que seus algoritmos aprendem com as informações que contém nos dados e não dependem de um 
fluxograma de passos como a programação clássica. Desta forma, os algoritmos de machine learning são capazes de identificar as regras (fluxograma) nos próprios dados, não dependendo de um programador especializado que as insira (Gutzwiller, et al, 2017), o que finalmente contribui para maior automatização do processo de predição dos parâmetros dos enlaces.

Neste trabalho, o projeto de enlaces ópticos são automatizados e otimizados por algoritmos de inteligência artificial onde são fornecidos dados de entrada como o tamanho do enlace, a taxa de transmissão de bits e a taxa de erro (BER) de bits aceitável e o algoritmo retornará a quantidade de amplificadores EDFAs, sua localização no enlace, potência de bombeamento e o comprimento da fibra de compensação de dispersão (DCF) que garantirá a BER desejada. Por se tratar de uma abordagem pioneira em projetos de links para comunicações ópticas, foram realizados estudos comparativos para identificar o algoritmo de inteligência artificial mais apto para otimizar o projeto de links ópticos, sendo utilizados os algoritmos redes Bayesianas, redes neurais perceptron (MLP), KNN, e rede neural Deep Learning.

\section{APLICAÇÕES DE INTELIGÊNCIA ARTIFICIAL EM COMUNICAÇÕES ÓPTICAS}

São observadas técnicas de inteligência artificial aplicadas em transmissão (Zibar, et al, 2015), controle de amplificação óptica (Huang, et al, 2016), monitoramento de rede (Qos) (Jiménez, et al, 2012), e mitigação de não-linearidades (Karinou, et al, 2017). Também são utilizados diferentes algoritmos de IA, tais como: rede Bayesianas (Zibar, et al, 2016), rede neural perceptron (Wu, et al, 2010) e rede neural deep learning, (Tanimura, et al, 2016) KNN (Wang, et al, 2016), SVM (Thrane, et al, 2017), K-means (Gonzalez et al, 2010). Sendo que os algoritmos SVM e K-means são aplicados na classificação do formato de modulação. (Mata, et al, 2018).

No escopo de inteligência artificial em projetos de links ópticos, o trabalho de Danish Rafique propõe prever o formato de modulação ideal para um enlace ao se utilizar de redes neurais (Rafique, 2018). No presente trabalho pretende-se uma otimização mais abrangente, predizendo com o uso de IA a quantidade de EDFAs e potência de bombeamentos desses amplificadores, como também sua localização e comprimento necessário para a fibra DCF, a fim de corrigir as distorções com o menor uso de equipamentos de regeneração. O pesquisador Darko Zibar realiza uma análise para avaliar os reais benefícios da inteligência artificial em comunicações ópticas, visando eliminar falsas expectativas e enfatizar o grande benefício de automatização que a IA tem oferecido para reduzir o custo humano na resolução de dilemas (Zibar et al, 2017).

Atualmente criação de softwares de programação clássica poderá enfrentar dificuldades na solução de melhorias e suporte tecnológico, dado que são muitas as variáveis que devem ser levadas em consideração na elaboração e construção de um sistema óptico. Assim, evidencia-se que a construção de um pseudocódigo de um software da programação clássica não seria trivial, logo se aponta a necessidade de um suporte "autônomo" para essa finalidade de redução do custo humano.

\section{METODOLOGIA}

O software VPIphotonics foi utilizado para simulação dos links ópticos, conforme apresentado na figura 1, para obter dados para alimentar o banco de dados utilizado para o treinamento dos algoritmos de machine learning. Os cenários testados envolvem um link óptico composto por um transmissor OOK/NRZ, fibras monomodos (SMF), segmentos de fibras de compensação de dispersão (DCF), amplificadores EDFAs e analisadores de sinais. Foram variados os parâmetros para extrair diversas situações distintas de enlaces ópticos, sendo eles: o comprimento total das fibras SMF (50 - $100 \mathrm{~km})$, número de amplificadores EDFAs (1 - 3), potência de bombeamento $(0,01-2,2575 \mathrm{~W})$, a taxa de bits do transmissor $(40-100 \mathrm{~GB} / \mathrm{s})$ e o comprimento da fibra de compensação de dispersão $(22-45 \mathrm{~km})$. O parâmetro de dispersão SMFs foi estabelecido em $18 \mu \mathrm{S} / \mathrm{m}^{2}$ e a sua inclinação de dispersão em $900 \mathrm{~S} / \mathrm{m}^{3}$. A dispersão dos DCFs foi fixada em $-40 \mu \mathrm{S} / \mathrm{m}^{2}$ e a sua inclinação de dispersão foi fixada em $117 \mathrm{~S} / \mathrm{m}^{3}$, de acordo com as fibras comercialmente disponíveis consultadas. Todos os outros parâmetros são o padrão do VPI. 
Os algoritmos de machine learning utilizados nesse estudo foram: redes Bayesianas, algoritmo KNN, rede neural MLP Perceptron e rede neural deep learning. Foram escolhidos esses algoritmos devido à suas aplicabilidades em comunicações ópticas conforme descrito na literatura. Para o treinamento dos algoritmos, foi necessário o uso de dois frameworks de código livre, o software WEKA, uma biblioteca de mineração de dados e aprendizado de máquina que fora aplicada no treinamento dos algoritmos KNN, Rede Bayesiana e MLP. Já a biblioteca $\mathrm{H} 2 \mathrm{O}$ de aprendizado de máquina e inteligência artificial foi necessária para o treinamento da rede neural Deep Learning. Os dados adquiridos na simulação computacional com o VPIphotonics foram inseridos em uma planilha eletrônica para normalização e rotulação dos dados. Sendo os rótulos dos dados a descrição dos equipamentos usados na simulação e a BER que cada um dos cenários alcançou.

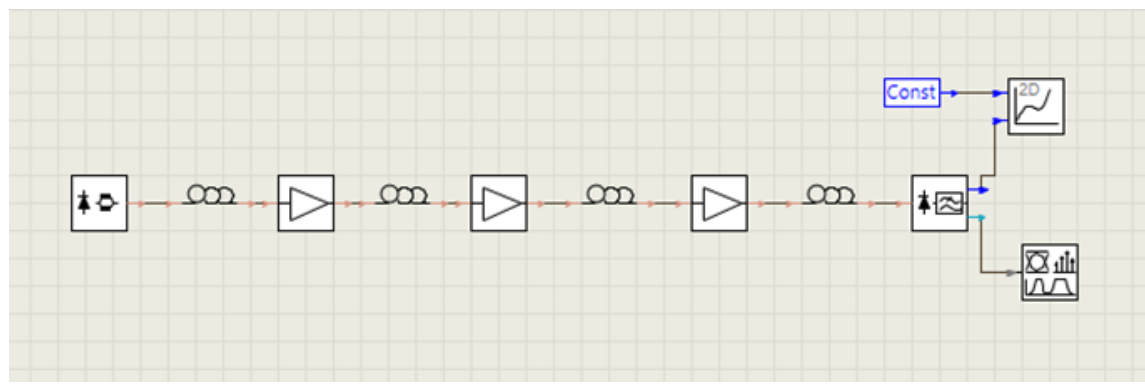

Figura 1. Captura do link óptico no simulador VPI (contendo emissor laser, EDFAs e analisadores de sinal)

\section{TREINAMENTO DOS ALGORITMOS}

Os algoritmos tentaram encontrar padrões e correlações nos dados, baseando-se nas variações dos parâmetros dos enlaces ópticos. Buscaram encontrar uma taxa de precisão alta na classificação posterior pelos algoritmos e aferir sua capacidade de entender o problema e prever uma resolução adequada. Havendo um total de 301 cenários distintos de links ópticos com suas respectivas BERs obtidas por simulações no VPIphotonics e inseridos nos algoritmos, todos os dados para o treinamento foram rotulados conforme o paradigma de aprendizado supervisionado adotado nesse trabalho.

\subsection{Algoritmo Rede Bayesiana}

O algoritmo Bayesiano usa o teorema de Bayes para realizar suas análises e classificações, como mostra a equação 1:

$$
\operatorname{prob}(\mathrm{B} \mid \mathrm{A})=(\operatorname{prob}(\mathrm{A} \mid \mathrm{B}) \operatorname{prob}(\mathrm{B})) / \operatorname{prob}(\mathrm{A})
$$

A taxa de precisão do algoritmo corresponde à capacidade da rede Bayesiana de classificar corretamente as configurações dos links ópticos ensinados ao algoritmo. Das 301 simulações informadas ao algoritmo, o mesmo alcançou $27 \%$ de precisão. Classificou corretamente 84 cenários e classificou incorretamente 217 cenários. O presente trabalho aborda um ambiente multiclasse, porém a rede Bayesiana enfrenta dificuldades em relacionar a correlação entre múltiplas variáveis. Ela ainda apresenta um gap de modelos que permitem aprofundar aplicações em seus resultados. (Figura 3).

\subsection{Algoritmo KNN}

O algoritmo K - Nearest Neighbors (KNN) analisa os dados medindo a distância euclidiana entre os dados recebidos, a fim de encontrar o número " $\mathrm{k}$ " de dados mais próximos do dado desconhecido, sendo ele um algoritmo de classificação não-linear. Foram inseridos 301 dados no treinamento e a taxa de precisão 
alcançada foi de $94 \%$, classificando corretamente 285 instâncias e incorretamente 16 instâncias. O algoritmo KNN demonstra robustez na análise de dados multivariados. O banco de dados inseridos no algoritmo contém grande variedade de variáveis e o método de classificação não-linear do algoritmo KNN o torna mais apto para abordagens envolvendo múltiplas variáveis, identificando padrões e correlações dispersas em comparação com algoritmos de classificação linearmente separáveis (Figura 3).

\subsection{Rede Neural Perceptron (MLP)}

A rede neural perceptron é um subcampo de inteligência artificial, onde funções matemáticas emulam o comportamento biológico do neurônio humano (Figura 2). A parametrização da MLP usada nesse trabalho abrange os seguintes escopos: duas camadas, sendo uma a camada de entrada com 100 "neurônios" e outra uma camada oculta com 150 "neurônios", uma taxa de aprendizado de 0,5 e um momentum de 0,3, com uma função de ativação sigmoide atingindo 1500 épocas de treinamento. Foram inseridos 301 dados e a MLP alcançou uma precisão de $24 \%$, classificando corretamente a 74 dados e incorretamente a 227 . A função de ativação sigmoide apresenta o dilema do gradiente pequeno, ou seja, tende a zero quando a saída não aprendeu, saturando o processo de aprendizado. Isso resulta em níveis de precisão menores. A função de ativação Softmax é mais adequada para problemas generalistas ou ainda um do tipo multiclasses como era o caso do estudo do presente trabalho. Fora optado o uso de uma taxa de aprendizado mais elevada $(0,5)$ para diminuir o número de épocas de treinamento, entretanto a MLP só conseguiu treinar com um mínimo de 1500 épocas de treinamento. (Figura 3).

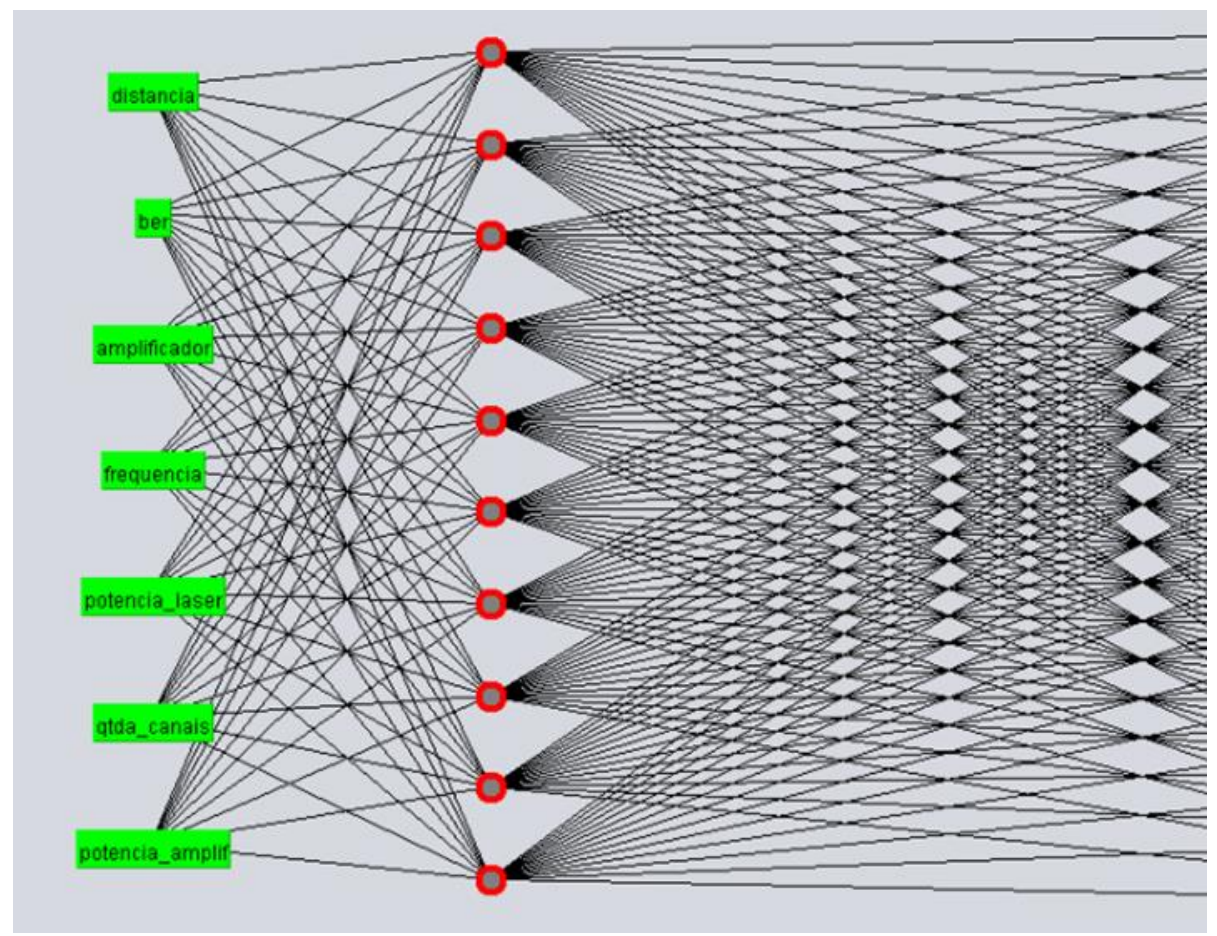

Figura 2. Rede neural MLP treinada no Weka

\subsection{Deep Learning}

Deep Learning é uma rede neural de múltiplas camadas ocultas, classificada como um subcampo da Machine Learning. A construção de uma rede neural de aprendizado profundo (Deep Learning) foi estabelecida pela biblioteca $\mathrm{H} 2 \mathrm{O}$ de código aberto, e quatro camadas foram utilizadas para a parametrização. A primeira camada é a de entrada e na sequência vêm duas camadas ocultas e uma camada de saída. A função de ativação utilizada foi a ReLU, a camada de entrada continha 300 neurônios, as camadas ocultas tinham 400 
neurônios cada uma e 300 neurônios totalizava a camada de saída. O número de épocas utilizado foi de 500, atingindo uma taxa de precisão de $99 \%$. Sua função de ativação ReLU não-linear se mostra uma função de redes neurais adequada para trabalhar em ambientes não-linearmente separáveis e com diversas variáveis, diferentemente da função de ativação sigmóide que sofre com um pequeno gradiente (Figura 3).

Treinamento dos algoritmos de machine learning

\begin{tabular}{lclc}
\hline Algoritmos & Acurácia & $\begin{array}{l}\text { Percentual de } \\
\text { Acerto }\end{array}$ & $\begin{array}{l}\text { Percentual de } \\
\text { Erro }\end{array}$ \\
\hline DEEP LEARNING & $99 \%$ & 300 & 1 \\
KNN & $94 \%$ & 285 & 16 \\
REDE BAYESIANA & $27 \%$ & 84 & 287 \\
REDE NEURAL (MLP) & $24 \%$ & 74 & 227 \\
& & & \\
\hline
\end{tabular}

Figura 3. Acurácia atingida pelos algoritmos no treinamento e valores de acertos e erros

A Deep Learning identificou as variáveis relevantes que influenciam nos resultados, observando que quanto maior a potência tanto maior será a chance de aparição de efeitos não-lineares. Também conclui que ao se aumentar a frequência, potencializa-se o surgimento de dispersão cromática. O tamanho da fibra DCF ideal compensa de forma precisa a dispersão, e esses parâmetros de mitigação do sinal trafegado foram aprendidos pela deep learning (Figura 4).

- importãncias variáveis

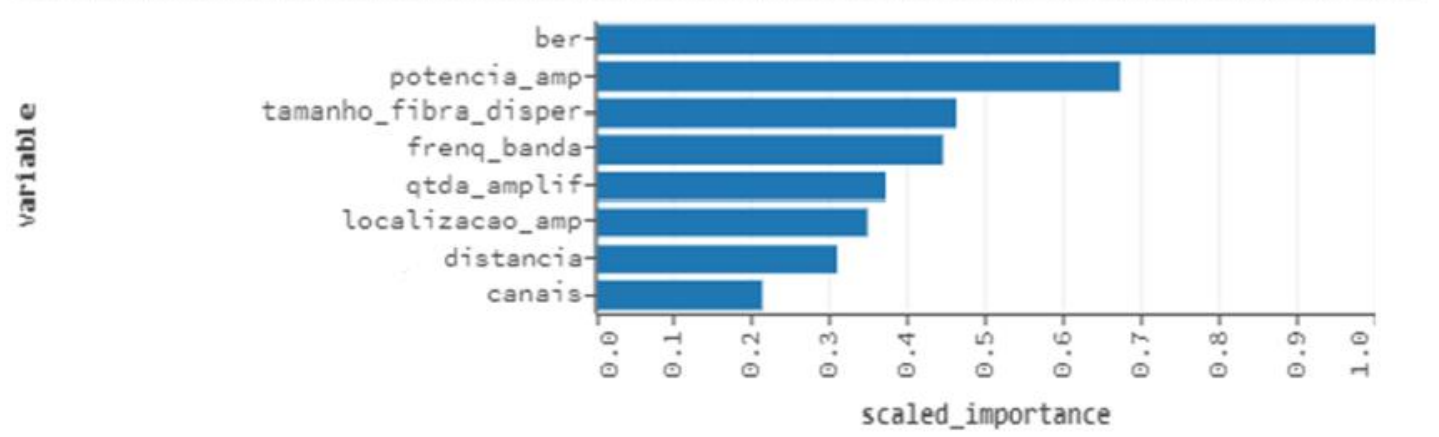

Figura 4. Gráfico de variáveis relevantes no treinamento da deep learning

\subsection{Predições dos Algoritmos}

Para realizar as predições, fora informado aos algoritmos o comprimento desejado do enlace óptico e a frequência de bits almejada. Desta forma os algoritmos devem retornar a quantidade de equipamentos de regeneração de sinal necessários para alcançar uma BER satisfatória $\left(<1 \times 10^{-9}\right)$ ou solicitada $\left(1 \times 10^{-10}\right)$. As configurações de enlace inseridas nos algoritmos foram as seguintes: $100 \mathrm{~km}$ de comprimento total, ora com 
$100 \mathrm{~Gb} / \mathrm{s}$ de taxa de transmissão de bits, ora com $40 \mathrm{~Gb} / \mathrm{s}$. O algoritmo KNN obteve o melhor desempenho de previsão, atingindo com suas predições a BER desejada. A Deep Learning apresentou performance semelhante ao KNN, entretanto com pequenas variações na BER idealizada. A rede Bayesiana e rede neural perceptron (MLP) não conseguiram predizer links com uma BER aceitável. A figura 5 mostra os resultados.

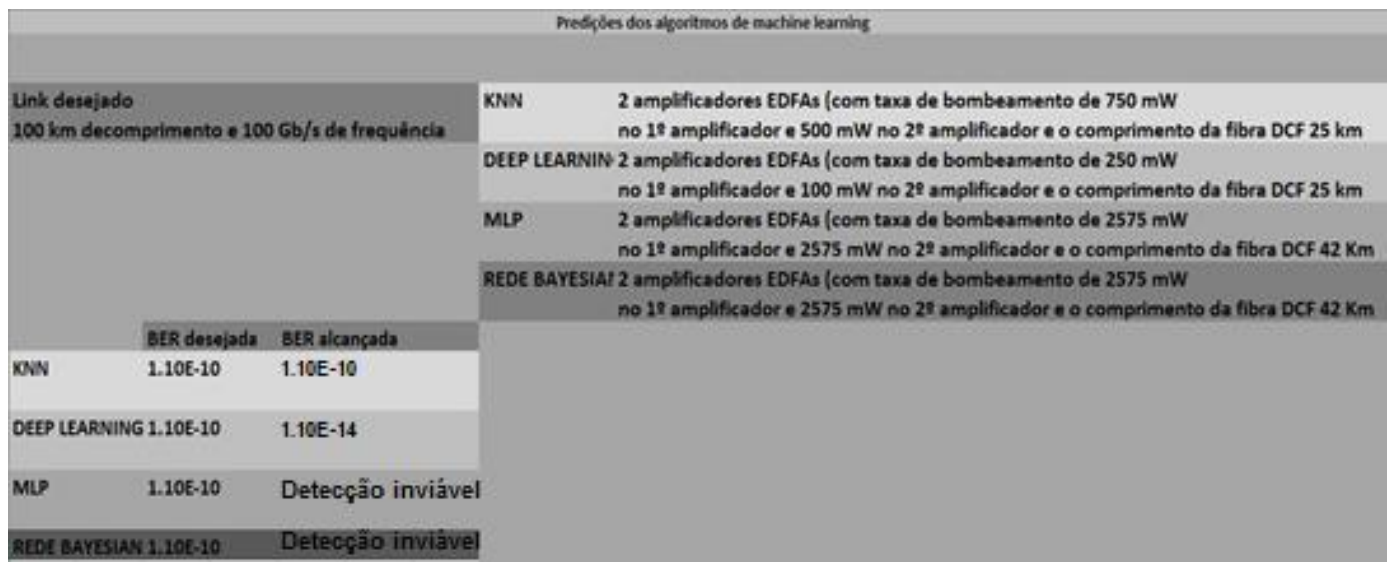

Figura 5. Resultados das predições dos algoritmos treinados (mostra o enlace solicitado aos algoritmos, a configuração que cada algoritmo retornou, e a BER solicitada e alcançada por cada um)

\section{CONCLUSÃO}

Os resultados obtidos são animadores, demonstrando a capacidade da inteligência artificial em aprender dilemas complexos e multifatoriais. Os algoritmos treinados aparentaram levar em conta o problema das distorções ópticas em suas predições e as manobras para amenizar seu impacto. Comparando-se os algoritmos utilizados, o algoritmo KNN apresentou melhor precisão e previsão. A técnica de rede neural Deep Learning obteve resultados semelhantes ao KNN. As redes Bayesiana e MLP apresentaram resultados inferiores e insatisfatórios, apontando uma dificuldade em ambientes com múltiplas variáveis. Tais técnicas podem contribuir para automação de projetos de links ópticos em paralelo, reduzindo o custo final dos projetos de links ópticos com mão de obra qualificada ao fazer uso mais inteligente dos recursos de rede com construção rápida (quando comparado com o delay humano). Evita-se, assim, paralisações do link pela manutenção corretiva ou a perdas das informações trafegadas. As direções futuras desse trabalho apontam para deixar o cenário de simulações mais realista do ponto de vista dos desafios da comunicação óptica, acrescentando canais ao enlace e adicionando-se formatos de modulação mais complexos (QAM, PSK). Paralelamente, tensiona-se usar o paradigma de aprendizado não-supervisionado, a fim de que os algoritmos possam encontrar padrões desconhecidos nas comunicações ópticas que sejam relevantes cientificamente. Por fim, dado que tecnologias como Big Data, IoT, computação em nuvem e mineração de dados estão apenas no começo, devem haver preparativos para a crescente demanda por conectividade, onde cientistas e governos terão que providenciar soluções inovadoras e eficientes que garantam um uso confortável para as tecnologias de comunicações digitais, onde a automação desponta como uma candidata para tal tarefa.

\section{AGRADECIMENTO}

Esse trabalho foi apoiado pela FAPESP (grants. $\mathrm{n}^{\circ}$ 2012/50259-8 e $\mathrm{n}^{\circ}$ 2015/11779-4), CNPq, e MackPesquisa. 


\section{REFERÊNCIAS}

Gonzalez N.G., D. Zibar, I.T. (2010) Monroy Cognitive digital receiver for burst mode phase modulated radio over fiber links Optical Communication (ECOC), 36th European Conference and Exhibition, IEEE, pp. 1-3.

Gutzwiller, R. S., Reeder, J. (2017) "Human interactive machine learning for trust in teams of autonomous robots". In: IEEE Conference on Cognitive and Computational Aspects of Situation Management. doi: 10.1109 / COGSIMA.2017.7929607

Huang C., M. Liu, S. Chu, C. Cheng. (2004) Application of machine learning techniques to web-based intelligent learning diagnosis system Fourth International Conference on Hybrid Intelligent Systems (HIS'04).

Huang Y., W. Samoud, C.L. Gutterman, C. Ware, M. Lourdiane, G. Zussman, P. Samadi, K. Bergman (2016) A machine learning approach for dynamic optical channel add/drop strategies that minimize EDFA power excursions. Proceedings of ECOC 2016; 42nd European Conference on Optical Communication, VDE, pp. 1-3.

Jiménez T. , JC Aguado , I. de Miguel , RJ Durán , N. Fernández, M. Angelou , D. Sánchez , N. Merayo , P. Fernández , N. Atallah, et al. (2012) A cognitive system for rapid transmission quality assessment in central optical networks Fiber Optic Communication Conference and Exhibition (OFC /

NFOEC), e Conferência Nacional de Engenheiros de Fibra Óptica, IEEE ( 2012 ), pp. 1 - 3.

Karinou F., N. Stojanovic, C. Prodaniuc, M. Agustin, J. Kropp, N.N. Ledentsov (2017) Solutions for 100/400-Gb/s ethernet systems based on multimode photonic technologies. J. Lightwave Technol., 35 (15), pp. 3214-3222.

Kyounghyun P., M. Chau Nguyen, H. Won. (2015) "Web-based collaborative big data analytics on big data as a service platform”. In: International Conference on Advanced Communication Technology. doi: 10.1109/ICACT.2015.7224859

Mata J., I. Miguel, R. Durán, N. Merayo, S. Singh, A. Jukan, M. Chamania. (2018) Artificial intelligence (AI) methods in optical networks: A comprehensive survey Optical Switching and Networking, Pages 43-57.

Rafique, D. (2018). Machine Learning Based Optimal Modulation Format Prediction for Physical Layer Network Planning. 1-4. Disponível: doi: 10.1109/ ICTON.2018.8473593

Shamsuddin, S. M.; Hasan, S. (2015) “Data science vs big data @ UTM big data centre”. In: International Conference on Science in Information Technology. doi: 10.1109 / ICSITech.2015.7407766

Tan, T. G.; Teo, J. ; Anthony, P.. (2010) "A simple heuristic search method for the automatic generation of neural-based game artificial intelligence architectures in Ms. PacMan”. In: International. doi: 10.1109/ ISSPA.2010.5605407

Tanimura T. , T. Hoshida, J.C. Rasmussen, M. Suzuki, H. Morikawa (2016) OSNR monitoring by deep neural networks trained with asynchronously sampled data 2016 21st OptoElectronics and Communications Conference (OECC) Held Jointly with 2016 International Conference on Photonics in Switching (PS), IEEE,pp.1-3.

ThraneJ., J. Wass, M. Piels, J.C. Diniz, R.Jones, D. Zibar (2017), Machine learning techniques for optical performance monitoring from directly detected PDM-QAM signals J. Lightwave Technol., 35 (4) pp. 868-875.

Wang D., M. Zhang, M. Fu, Z. Cai, Z. Li, Y. Cui, B. Luo (2016) KNN-based detector for coherent optical systems in presence of nonlinear phase noise 2016 21st OptoElectronics and Communications Conference (OECC) Held Jointly with 2016 International Conference on Photonics in Switching (PS), IEEE, pp. 1-3.

Wu X., J.A. Jargon, R.A. Skoog, L. Paraschis, A.E. Willner (2010) Applications of artificial neural networks in optical performance monitoring J. Lightwave Technol., 27 (16) (2009), pp. 3580-3589 Conference on Information Science, Signal Processing and their Applications.

Zhao C. Hongwei C. Quanchun L (2017) "Bayesian algorithm-based traffic prediction of big data services in OpenFlow controlled optical networks". In: IEEE 2nd International Conference on Big Data Analysis. doi: 10.1109 / ICBDA.2017.8078752

Zibar D., L.H.H. de Carvalho, M. Piels, A. Doberstein, J. Diniz, B. Nebendahl, C. Franciscangelis, J. Estaran, H. Haisch, N.G. Gonzalez, et al. (2015) Application of machine learning techniques for amplitude and phase noise characterization. J. Lightwave Technol., 33 (7), pp. 1333-1343.

Zibar D., M. Piels, R. Jones, C.G. Schäeffer. (2016) Machine learning techniques in optical communication J. Lightwave Technol., 34 (6), pp. 1442-1452.

Zibar D., Wymeersch H., Lyubomirsky I. (2017) Machine learning under the spotlight Nature Photonics volume 11, pages749-751. 